\title{
Structural brain abnormalities in cervical dystonia
}

\author{
Tino Prell ${ }^{1 *}$, Thomas Peschel ${ }^{2}$, Bernadette Köhler ${ }^{3}$, Martin H Bokemeyer ${ }^{4}$, Reinhard Dengler ${ }^{3}$, \\ Albrecht Günther ${ }^{1,5}$ and Julian Grosskreutz ${ }^{1}$
}

\begin{abstract}
Background: Idiopathic cervical dystonia is characterized by involuntary spasms, tremors or jerks. It is not restricted to a disturbance in the basal ganglia system because non-conventional voxel-based MRI morphometry (VBM) and diffusion tensor imaging (DTI) have detected numerous regional changes in the brains of patients.

In this study scans of 24 patients with cervical dystonia and 24 age-and sex-matched controls were analysed using VBM, DTI and magnetization transfer imaging (MTI) using a voxel-based approach and a region-of-interest analysis. Results were correlated with UDRS, TWSTRS and disease duration.
\end{abstract}

Results: We found structural alterations in the basal ganglia; thalamus; motor cortex; premotor cortex; frontal, temporal and parietal cortices; visual system; cerebellum and brainstem of the patients with dystonia.

Conclusions: Cervical dystonia is a multisystem disease involving several networks such as the motor, sensory and visual systems.

Keywords: Visual system, Basal ganglia, Dorsolateral prefrontal cortex

\section{Background}

Idiopathic cervical dystonia, the most common form of focal dystonia [1], is characterized by involuntary posturing of the head caused by involuntary spasms, tremors or jerks, and it is frequently accompanied by neck pain. Typical patterns are the rotational torticollis, laterocollis and retro- or antecollis [2]. Diagnosis is based on history, neurological examination and exclusion of other causes. Functional and structural neuroimaging techniques have detected regional activation and structural changes in the brains of patients with cervical dystonia.

Till date, 5 studies have used voxel-based morphometry (VBM) as a fully automated, operator-independent, wholebrain image analysis technique to evaluate cervical dystonia. They revealed an increase in grey matter volume in the internal globus pallidus, thalamus, cerebellum, motor cortex and supplementary motor area. However, the results are conflicting because a decrease in grey matter was also observed in the putamen, supplementary motor area, right visual cortex and right dorsal lateral prefrontal cortex [3-6].

\footnotetext{
* Correspondence: tino.prell@med.uni-jena.de

${ }^{1}$ Hans-Berger Department of Neurology, Jena University Hospital, Erlanger Allee 101, 07747 Jena, Germany

Full list of author information is available at the end of the article
}

Diffusion tensor imaging (DTI), which is more sensitive to pathology in white matter fibres, detects alterations in the degree (diffusivity, ADC) and directedness (fractional anisotropy, FA) of proton movement, which reflect microstructural tissue changes and fibre organisation. Several studies have examined DTI measures in patients with different forms of dystonia. Similar to VBM studies, the results of DTI studies are contradictory. In patients with hereditary dystonia, FA was decreased in the sensorimotor cortex [7]. In an ROI-based study of patients with dystonia, FA was decreased in the corpus callosum and increased in the putamen $[8,9]$. Diffusivity was enhanced in the prefrontal cortex and decreased in the caudate nucleus and putamen [9]. In contrast, in another ROI study, diffusivity in the pallidum, putamen and caudate nucleus was increased in patients with cervical dystonia $[6,10]$.

Magnetization transfer imaging (MTI) detects the relative proportion of free mobile protons and immobile protons bound to macromolecules, i.e. in myelin and axonal loss $[11,12]$, and it has not yet been applied to patients with dystonia.

We aimed to use a voxel-based analysis and an ROIbased approach to evaluate idiopathic cervical dystonia and extend previous MRI studies. Voxel-based analysis is a technique that can identify microstructural changes in any part of the whole brain without a prior hypothesis, 
unlike ROI-based studies. We therefore studied a moderate sample of 24 patients with dystonia using VBM, DTI and MTI.

\section{Methods}

\section{Subjects}

Twenty-four patients (18 females, 6 males) with idiopathic cervical dystonia (mean age, 52 years) and 24 age- and sex-matched controls (18 females, 6 males; mean age, 53 years, $\mathrm{p}>0.05$ ) from the Medical School Hannover were enrolled in this prospective study from July 2004 until March 2005. Patients who were diagnosed with idiopathic cervical dystonia by a neurologist experienced in the diagnosis of dystonia were included. Exclusion criteria were as follows: presence of any multifocal dystonia with blepharospasm or oromandibular manifestations, a positive family history for dystonia and a history of any other neurological disease or relevant vascular diseases. Every patient was regularly treated with botulinum toxin, and the last injection was administered 4 weeks before MRI imaging. Patients were evaluated using the Unified Dystonia Rating Scale (UDRS) and Toronto Western Spasmodic Torticollis Rating Score (TWSTRS). Using the Mini-Mental Status Examination (MMSE) and the Beck Depression Inventory (BDI), clinically relevant dementia or depression was ruled out (Table 1). Exclusion criteria for healthy voluntary controls were: history of neurological or psychiatric disorders, vascular diseases (e.g. stroke), diabetes mellitus or the intake of psychoactive drugs. This study was approved by the local ethics committee of the Hannover Medical School, and all subjects provided written informed consent.

\section{Data acquisition}

Images were acquired on a neuro-optimized 1.5-T GE Signa Horizon NV/i (General Electric Company, Milwaukee, WI, USA) using a 3-dimensional, T1weighted, spoiled gradient-recalled echo (SPGR) sequence generating 124 contiguous sagittal slices (RT, $24 \mathrm{~ms}$; TE, $8 \mathrm{~ms}$; flip angle, $180^{\circ} ; 2$ averages; acquisition time, 13'10"; in-plane resolution, $0.97 \times 0.97 \times 1.5 \mathrm{~mm}^{3}$ ). The protocol for MTI included a proton density (PD)weighted spin echo sequence (TR, 2600; TE, 20; $256 \times$ 256), both with (MT) and without (non-MT) a preparing saturation pulse $\left(1200 \mathrm{~Hz}\right.$ off-resonance, $1180^{\circ}$ flip-angle, $16 \mathrm{~ms}$ ). Forty-three slices of 3-mm thickness aligned along the $\mathrm{AC}-\mathrm{PC}$ line were acquired. Image post-processing

Table 1 Disease characteristics of probands

\begin{tabular}{cccccccc}
\hline & $\begin{array}{c}\text { Sex } \\
(\mathbf{f} / \mathbf{m})\end{array}$ & $\begin{array}{c}\text { Age } \\
(\mathbf{y})\end{array}$ & UDRS & TWSTRS & $\begin{array}{c}\text { Disease duration } \\
(\mathbf{y})\end{array}$ & BDI & MMST \\
\hline Patients & $18 / 6$ & 52 & 4.3 & 22.5 & 13.9 & 7,5 & 28.3 \\
Controls & $18 / 6$ & 53 & - & - & - & - & 29.1 \\
\hline
\end{tabular}

included a simple intersequence correction of movement with the automated image registration package based on the rigid body model (AIR) [13] and calculation of MTR maps pixel-by-pixel according to the following formula: MTR $=[($ non-MT $-\mathrm{MT}) /$ non-MT $] \times$ 100. During scanning, all participants were comfortably placed, and their heads were fixated within the headcoil with special cushions.

\section{Pre-processing of structural data}

Data were processed on a standard IBM-compatible PC using SPM2 statistical parametric mapping software (Welcome Department of Cognitive Neurology, London), and they were worked on in an analysis environment (MATLAB; the Math Works Inc, Natick, Mass). The images were re-oriented into oblique axial slices aligned parallel to the anterior-posterior commissural axis with the origin set to the anterior commissure.

\section{Data pre-processing for VBM}

An optimized version of the VBM protocol was followed as described by our group [14]. The resulting images were re-sliced to a final voxel size of $1 \mathrm{~mm}^{3}$. Voxel values in segmented images of grey and white matter were multiplied by the Jacobian determinants derived from spatial normalization to provide an intensity correction for induced regional volumetric changes, thus preserving within-voxel volumes that may have been altered during non-linear normalization. These 'modulated' images were smoothed to $8 \mathrm{~mm}$ using a full width half-maximum (FWHM) Gaussian filter to minimize individual gyral variations and increase the statistical validity of the analysis.

\section{Data pre-processing for voxel-based DTI}

After calculation of the FA and ADC maps, images were pre-processed using an approach adopted from VBM as described by our group [14]. This included an optimized normalization procedure, automated removal of skull and CSF signals as well as smoothing (8-mm FWHM). All EPI scans were normalized to the EPI template provided by SPM and further used to create a site-specific EPI template appropriate to the population sample with scanner-specific image contrast. This site-specific template was used again for normalization and brain extraction for the individual images in the group studied, thus resulting in optimal normalization and cleaning parameters for use with the FA and ADC images.

\section{Data pre-processing for voxel-based MTI}

The main challenge of voxel-based MTI analysis involves meeting the requirement for an optimal matching of the brains being compared. Therefore, a complex preprocessing procedure was employed as previously described [15]; this procedure included the creation of a 
series of templates in order to derive the best possible parameter set for normalization. To achieve maximum precision, a proton density (PD)-weighted template with scanner-specific image contrast best adapted for the studied sample population was created. Therefore, all PD-weighted scans were normalized to the PD template provided by SPM2 and subsequently smoothed with an 8-mm isotropic FWHM isotropic Gaussian kernel. Therefore, a mean image was created that served in the following as the sample population and scanner specific template, and all PD-images in native space were normalized to it. To minimize the influence of structures other than the brain on registration, the normalized images were skull-stripped using the segmentation procedures implemented in SPM2, which included the brain extraction step. The resultant white and grey matter partitions were summed and set at a threshold of 0.15 , thus discarding the majority of extracerebral tissue and CSF while preserving the original voxel intensities. The obtained normalized skull-stripped images were then smoothed with an 8-mm isotropic FWHM Gaussian kernel, and a new skull-stripped PD-weighted template was created. The PD images in native space (non-normalized) were subject to exactly the same procedure, wherein they were skull-stripped, segmented, multiplied with a binary mask and normalized to the previously skull-stripped PDweighted template. This prevented any contribution of non-brain voxels, afforded optimal spatial normalization of the individual PD images and provided an optimized normalization parameter set for the PD images adapted to the investigated population sample. Because the segmentation of native images is performed on affine-normalized images, and because the probability maps used as Bayesian priors for segmentation are in stereotactic space, the optimized normalization parameter set was re-applied to the original PD-weighted images in native space. These optimally normalized PD images-now in stereotactic space-were again skull-stripped using the above described procedure. This resulted in optimally normalized PD images removed from extracerebral tissue and CSF. Finally, the optimized normalization parameter set was applied to the inherently co-registered MTR maps in native space and re-sliced with a final voxel size of $1 \mathrm{~mm}^{3}$. The normalized MTR images were then skullstripped by applying the corresponding brain mask derived from the optimally normalized PD-weighted images. Analogous to the PD images, this resulted in optimally normalized MTR maps removed from non-brain structures. Images were smoothed to $8 \mathrm{~mm}$ using an FWHM Gaussian filter to improve signal-to-noise ratio.

\section{Statistical analysis}

Processed images of each tissue class were analyzed in the framework of the general linear model. For VBM, group comparison of patients with dystonia and healthy controls was performed in SPM2 using the model 'compare-populations, one scan/subject (ANCOVA)'. During modulation, we incorporated the correction for volume change induced by spatial normalization. Therefore, it was appropriate to include the global mean voxel value of each tissue class as a covariate to determine the regionally specific pattern of loss or gain within each compartment and eliminate any variance due to differences in head size. Regression analyses with clinical measures were explored for the patients using the SPM2 model 'simple regression (correlation)'. With regard to group comparisons, ANCOVA with the mean voxel value was used to normalize image intensity in the different tissue maps to allow for identification of the regional pattern of these correlations. MTR maps were analyzed separately using the model 'compare-populations, one scan/ subject (two sample $t$-test)'. Regression analyses with clinical measures in the patients were explored using the model 'simple regression (correlation)'. The resulting statistical parametric maps of VBM and MTR were derived at a significance level of $\mathrm{p}<0.001$, uncorrected with a threshold of 100 voxels.

In the group comparison, a small volume correction (SVC) limited to the volume of that particular region was performed for regions in which an effect was hypothesized, namely the globus pallidum and visual cortex [3-5,16], using a sphere with a radius of $15 \mathrm{~mm}$ [17]. Here we controlled for multiple comparisons using the family-wise error (FWE) method $(\mathrm{p}<0.05)$.

Moreover, for the group comparison, region of interest (ROI) analyses were performed using MarsBar (MARSeille Boîte À Région d'Intérêt; release 0.43) and the AAL ROI archive, which is a toolbox for SPM providing routines and an ROI library (http://marsbar.sourceforge.net/). The ROIs were as follows: cerebellum, occipital lobe, para/hippocampal region, frontal lobe, parietal lobe, temporal lobe and precuneus. The ROI definitions are described in the study by Tzourio-Mazoyer et al. [18].

\section{Results}

\section{Clinical characterization}

Twenty-four patients with dystonia (mean age 52 years) were enrolled in this study. The mean disease duration was 13.4 years (SD 10.4) and the mean age at disease onset was 37.1 years (SD 15.2). The mean TWSTRS was 22.5 points (SD 10.7). The subscores of TWSTRS were as follows. First, the mean Torticollis Severity Scale was 9.5 (SD 5.2). Maximal Excursion was rotation and laterocollis, and none of the patients had antercollis. Most of the patients had an intermittent $(25 \%-50 \%$ of the time) or a frequent deviation (50\%-75\%). Sensory tricks were completely or partially effective in $87.5 \%$ patients. Sixty-seven percent patients were able to maintain 
their head within $10^{\circ}$ of the neutral position without using sensory tricks for over 46 seconds. Second, the Mean Disability Scale was 6.2 points (SD 5.2). Third, the Mean Pain Scale was 6.4 (SD 3.7). The mean severity of pain was 3.2 , calculated as [worst + best $+(2 *$ usual $) / 4$ ] On an average, pain was present $26 \%-50 \%$ of the time, and it was a major contributor to disability in $42 \%$ patients. The mean UDRS was 4.25 (SD 4.1). The clinical parameters are summarized in Table 1.

\section{Group comparison}

\section{VBM}

In the voxel-based whole brain analysis, the dystonia patients displayed enhanced volume bilaterally in the lentiform nucleus (left globus pallidus, right claustrum, right putamen), left frontal eye field (BA 8) and the bilateral medial surface of the occipital lobe (visual cortex, BA 17) (Figure 1). In addition, there were regions with decreased grey matter volume, particularly in the left precentral gyrus, the left supplementary motor area (BA 6), the right somatosensory association cortex (BA 7) and the left medial temporal gyrus (Table 2, Figure 1). In the white matter tissue class and CSF, no significant voxels were found.

ROI analysis displayed an increase in grey matter volume in the visual system on the lateral surface of the occipital lobe (superior and middle occipital gyri), the lateral and medial surface of the parietal lobe (supramarginal and angular gyri, precuneus) and the limbic system (hippocampal and parahippocampal regions; Table 3). Decreased grey matter volume was not observed in the studied ROIs.

\section{MTI}

In the voxel-based whole brain analysis, MTR was enhanced in the temporal (left medial temporal gyrus, right temporal lobe BA 21) and parietal (left secondary somatosensory cortex BA 13) lobe of the patients with dystonia. Furthermore, MTR was increased in the left cingulate gyrus. MTR was decreased in the left dorsolateral prefrontal and frontal cortex (BA 9, 10, 11) and the bilateral primary and secondary visual cortices (BA 17, 19; Table 2, Figure 2).

\section{VBM patients $>$ controls}
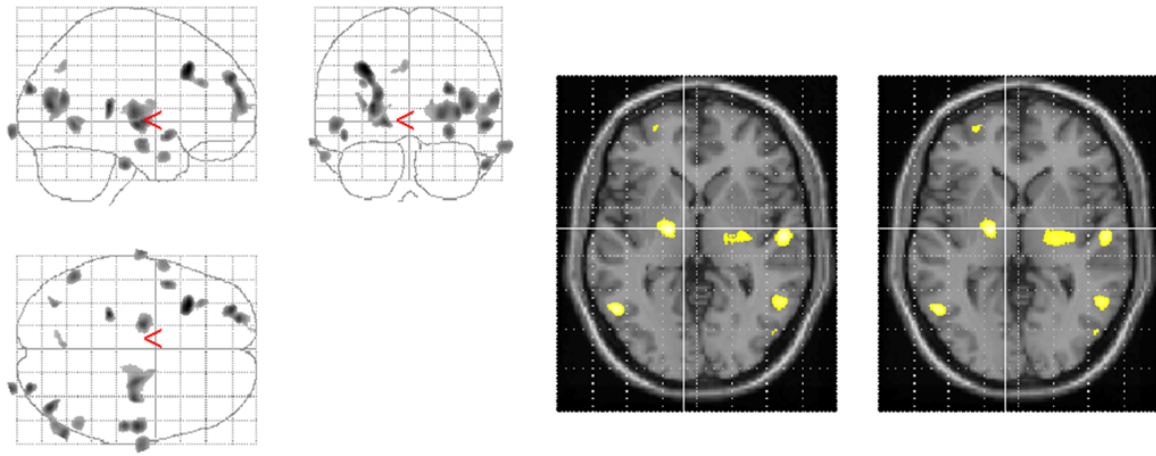

Tvalue

\section{VBM controls > patients}


Figure 1 Changes of grey matter volume in patients with dystonia compared to those in healthy controls (group comparison). VBM showed regional enhanced grey matter in the lentiform nucleus, the frontal eye fields (BA 8), and the secondary visual cortex (displayed at $p=$ 0.001, uncorrected, extent threshold 100 voxels). Grey matter atrophy was observed in the precentral gyrus, the supplementary motor area, the somatosensory association cortex, and in the medial temporal gyrus. The color bar represents the T-score. The differences between the groups are superimposed on a standard normalized T1-weighted image. Images are shown in neurological convention. 
Table 2 Changes of grey matter volume, MTI, and DTI-values in patients with dystonia compared to those in healthy controls (group comparison)

\begin{tabular}{|c|c|c|c|c|c|c|c|c|}
\hline \multirow[t]{2}{*}{ Brain region } & \multirow[t]{2}{*}{ Side } & \multirow[t]{2}{*}{ BA } & \multicolumn{3}{|c|}{ MNI space } & \multirow[t]{2}{*}{ t-value } & \multirow[t]{2}{*}{ P (uncorr.) } & \multirow[t]{2}{*}{ Cluster size } \\
\hline & & & $\mathbf{x}$ & $y$ & $\mathbf{z}$ & & & \\
\hline \multicolumn{9}{|c|}{ VBM } \\
\hline \multicolumn{9}{|c|}{ Grey matter volume increase } \\
\hline Frontal gyrus & left & 8 & -32 & 23 & 38 & 4.64 & 0.002 & 582 \\
\hline \multirow[t]{3}{*}{ Frontal gyrus } & left & 10 & -29 & 55 & 32 & 4.01 & 0.009 & 464 \\
\hline & & & -22 & 60 & 19 & 4.02 & & \\
\hline & & & -23 & 61 & 14 & 3.68 & & \\
\hline Medial globus pallidus (internal) & left & - & -17 & -9 & 0 & 3.99 & 0.011 & 147 \\
\hline \multirow[t]{2}{*}{ Claustrum, Putamen } & right & - & 35 & -16 & 7 & 3.90 & 0.014 & 640 \\
\hline & & & 24 & -15 & 7 & 3.83 & & \\
\hline Middle temporal gyrus & right & 39 & 42 & -76 & 12 & 4.06 & 0.009 & 454 \\
\hline Occipital lobe, cuneus & right & 19 & 28 & -90 & 23 & 4.07 & 0.031 & 118 \\
\hline Occipital lobe, cuneus & left & 19 & -53 & -58 & -3 & 3.71 & 0.022 & 149 \\
\hline \multicolumn{9}{|c|}{ Grey matter volume decrease } \\
\hline Precentral gyrus & left & 4 & -22 & -23 & 65 & 5.03 & 0.001 & 512 \\
\hline \multirow[t]{2}{*}{ Middle frontal gyrus } & left & 6 & -25 & -2 & 54 & 3.83 & 0.016 & 188 \\
\hline & & & -8 & -14 & 59 & 3.87 & 0.015 & 100 \\
\hline Parietal Lobe. Precuneus & right & 7 & 26 & -56 & 49 & 3.93 & 0.013 & 167 \\
\hline Middle temporal gyrus & left & 39 & -38 & -60 & 20 & 3.78 & 0.018 & 192 \\
\hline \multicolumn{9}{|c|}{ MTI } \\
\hline \multicolumn{9}{|c|}{ Increase of MTR } \\
\hline \multirow[t]{2}{*}{ Middle temporal gyrus } & left & 39 & -47 & -69 & 28 & 5.02 & 0.025 & 1854 \\
\hline & & 19 & -51 & -61 & 17 & 3.95 & & \\
\hline \multirow[t]{2}{*}{ Temporal lobe } & right & 21 & 42 & -4 & -23 & 4.47 & 0.004 & 2676 \\
\hline & & & 49 & -19 & -17 & 4.36 & & \\
\hline Inferior parietal lobule & left & 13 & -58 & -42 & 22 & 4.24 & 0.006 & 485 \\
\hline Cingulate gyrus & left & 31 & -6 & -47 & 42 & 3.86 & 0.016 & 104 \\
\hline \multicolumn{9}{|c|}{ Decrease of MTR } \\
\hline \multirow[t]{2}{*}{ Frontal gyrus } & left & 11 & -1 & 58 & -17 & 4.4 & 0.004 & 450 \\
\hline & & 10 & -35 & 38 & 26 & 4.23 & 0.006 & 612 \\
\hline Precentral gyrus & left & 9 & -36 & 23 & 39 & 4.10 & 0.009 & 423 \\
\hline Lingual gyrus & left & 17 & -15 & -95 & -12 & 4.17 & 0.007 & 730 \\
\hline Middle temporal gyrus & right & 19 & 40 & -75 & 18 & 4.14 & 0.008 & 246 \\
\hline \multicolumn{9}{|c|}{ DTI } \\
\hline \multicolumn{9}{|c|}{ Increase of FA } \\
\hline Middle frontal gyrus & left & 6 & -3 & -16 & 62 & 4.05 & 0.012 & 149 \\
\hline Brainstem. Pons & left & - & -13 & -29 & -29 & 3.94 & 0.016 & 213 \\
\hline Thalamus (Ncl. VPN, VPL) & left & - & -9 & -24 & -3 & 3.91 & 0.017 & 319 \\
\hline Middle temporal gyrus & left & 39 & -40 & -62 & 18 & 4.92 & 0.001 & 481 \\
\hline Posterior cingulate gyrus & right & - & 18 & -58 & 14 & 3.66 & 0.031 & 103 \\
\hline \multicolumn{9}{|c|}{ Decrease of FA } \\
\hline Precentral gyrus & right & 6 & 50 & 1 & 33 & 4.85 & 0.001 & 481 \\
\hline Postcentral gyrus & left & 3 & -38 & -25 & 50 & 4.23 & 0.008 & 582 \\
\hline
\end{tabular}




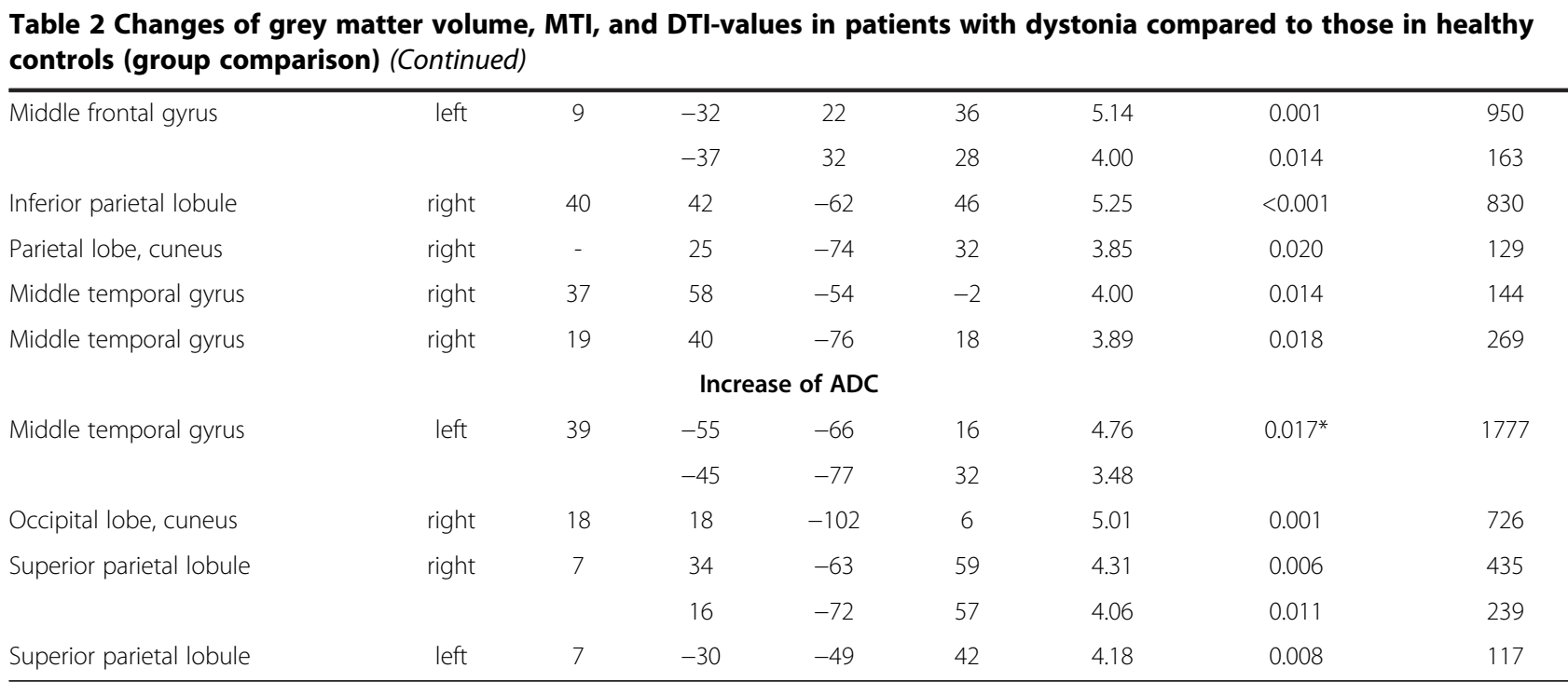

* significant with FDR (false discovery rate).

ROI analysis supported the involvement of the parietal lobe and, in particular, the inferior parietal lobule, by enhanced MTR. Similarly, in the lateral surface of the occipital lobe (superior and middle occipital gyri) and the limbic system (hippocampal and parahippocampal regions), MTR was found to be enhanced (Table 3). A decreased MTR was not observed in the studied ROIs.

\section{DTI}

FA values were changed in the motor, sensory, limbic and visual networks of the patients with dystonia. FA was enhanced in the supplementary motor area (middle frontal gyri, BA 6), pontine brainstem (medial lemniscus), thalamus (ventral posterior medial and lateral nucleus, VPM and VPL), middle temporal gyrus and cingulate gyrus (Table 2, Figure 3). FA was decreased in the precentral and postcentral gyri, dorsolateral prefrontal cortex and right secondary visual cortex (BA 19; Table 2, Figure 3).

Diffusivity was enhanced in the middle temporal gyrus (BA 19), medial surface of the occipital lobe (cuneus) and bilateral secondary somatosensory cortex (BA 7). Decreased diffusivity was not observed (Table 2, Figure 3 ).

ROI analysis showed enhanced FA in the parietal lobe and the limbic (hippocampal and parahippocampal regions) and visual systems (occipital lobe). A decreased FA was not observed. Similar to the whole brain approach, diffusivity was enhanced in the middle temporal gyrus (BA 19) and superior parietal lobule (Table 3).

\section{Correlation with clinical data}

Next, patient's clinical data such as disease severity and disease duration were correlated with VBM, MTI and DTI values. SVC and ROI analysis were not undertaken for studying these correlations.
The UDRS showed a clear positive correlation with grey matter decrease in the frontal areas, cingulate gyrus and inferior frontal gyrus, indicating that the progression of symptoms was reflected in an increase in grey matter volume in these areas. UDRS and MTR did not correlate significantly. With regard to DTI, UDRS showed a positive correlation with FA in the visual cortex (bilateral occipital lobe), cingulum and frontal and parietal lobes (BA 7). UDRS correlated negatively with ADC in the occipital lobe and cingulum (Table 4).

TWSTRS did not correlate with grey matter volume, MTR, FA and ADC.

The disease duration was not reflected in a specific pattern of volume decrease, but it correlated negatively with MTR in the primary motor cortex (BA 4), supplementary motor cortex (BA 6) and the right fusiform gyrus (BA 37). This indicates that neuronal density in the grey matter and myelinisation in the white matter decreases with increasing disease duration. The disease duration showed a negative correlation with FA in the temporal and parietal lobes, frontal eye field (BA 8) and the primary visual cortex. Disease duration and ADC correlated positively in the right visual cortex (BA 18) and negatively in the secondary somatosensory cortex (BA 40), precentral and postcentral gyri (BA 3, 4), supplementary motor cortex (BA 6) and the left frontal eye field (BA 8; Table 4).

\section{Discussion}

In the present study, structural changes in the brains of a moderate sample of dystonia patients were investigated by VBM, DTI and MTI. We found widespread alterations in grey and white matter tissue in the basal ganglia and the somatosensory, motor, limbic and visual systems, providing further evidence that dystonia is a 
Table 3 ROI analyses of grey matter volume, MTI, and DTI-values in patients with dystonia compared to those in healthy controls (group comparison)

\begin{tabular}{cccc}
\hline ROI & t-value $P$ (uncorr.) \\
\hline VBM
\end{tabular}

Grey matter volume increase

$\begin{array}{lccl}\text { Angular gyrus } & \text { right } & 2.75 & 0.004 \\ \text { Cerebellum } & \text { right } & 1.70 & 0.047 \\ \text { Inferior frontal gyrus, orbital part } & \text { right } & 1.81 & 0.038 \\ \text { Hippocampus } & \text { left } & 2.06 & 0.022 \\ \text { Middle occipital gyrus } & \text { right } & 2.32 & 0.012 \\ \text { Superior occipital gyrus } & \text { left } & 1.74 & 0.044 \\ \text { Superior occipital gyrus } & \text { right } & 2.43 & 0.009 \\ \text { Parahippocampal gyrus } & \text { left } & 2.23 & 0.015 \\ \text { Parahippocampal gyrus } & \text { right } & 1.93 & 0.029 \\ \text { Inferior parietal gyrus } & \text { left } & 2.13 & 0.019 \\ \text { Inferior parietal gyrus } & \text { right } & 2.25 & 0.014 \\ \text { Superior parietal gyrus } & \text { left } & 2.90 & 0.002 \\ \text { Superior parietal gyrus } & \text { right } & 2.81 & 0.003 \\ \text { Postcentral gyrus } & \text { left } & 2.15 & 0.018 \\ \text { Postcentral gyrus } & \text { right } & 1.58 & 0.060 \\ \text { Precentral gyrus } & \text { right } & 1.76 & 0.042 \\ \text { Precuneus } & \text { left } & 2.39 & 0.010 \\ \text { Precuneus } & \text { right } & 1.73 & 0.044 \\ \text { Supramarginal gyrus } & \text { right } & 2.07 & 0.021 \\ \text { Middle temporal gyrus } & \text { right } & 2.23 & 0.015 \\ \text { Cerebellum, vermis } & & 1.77 & 0.041\end{array}$

Grey matter volume decrease

none

MTR

Increase of MTR

Angular gyrus
Cerebellum 3
Inferior frontal gyrus, orbital
Hippocampus
Middle occipital gyrus
Superior occipital gyrus
Superior occipital gyrus
Parahippocampal gyrus
Parahippocampal gyrus
Paracentral lobule
Paracentral lobule
Inferior parietal gyrus
Inferior parietal gyrus
Superior parietal gyrus

$\begin{array}{cccl}\text { right } & 2.75 & 0.004 & \\ \text { right } & 1.70 & 0.047 & \\ \text { right } & 1.81 & 0.038 & \text { Angular gyrus } \\ \text { left } & 2.06 & 0.022 & \text { Cerebellum } \\ \text { right } & 2.32 & 0.012 & \text { Inferior frontal gyrus, orbital part } \\ \text { left } & 1.74 & 0.044 & \text { Hippocampus } \\ \text { right } & 2.43 & 0.009 & \text { Superior occipital gyrus } \\ \text { left } & 2.23 & 0.015 & \text { Superior occipital gyrus } \\ \text { right } & 1.93 & 0.029 & \text { Parahippocampal gyrus } \\ \text { left } & 1.83 & 0.037 & \text { Parahippocampal gyrus } \\ \text { right } & 2.06 & 0.022 & \text { Paracentral lobule } \\ \text { left } & 2.13 & 0.019 & \text { Paracentral lobule } \\ \text { right } & 2.25 & 0.014 & \text { Inferior parietal gyrus } \\ \text { left } & 2.90 & 0.002 & \text { Inferior parietal gyrus }\end{array}$

Table 3 ROI analyses of grey matter volume, MTI, and DTI-values in patients with dystonia compared to those in healthy controls (group comparison) (Continued)

\begin{tabular}{lccl}
\hline Superior parietal gyrus & right & 2.81 & 0.003 \\
Postcentral gyrus & left & 2.15 & 0.018 \\
Precentral gyrus & right & 1.76 & 0.042 \\
Precuneus & left & 2.39 & 0.010 \\
Precuneus & right & 1.73 & 0.044 \\
Supramarginal gyrus & right & 2.07 & 0.021 \\
Middle temporal gyrus & right & 2.23 & 0.015 \\
Cerebellum, vermis & & 1.77 & 0.041
\end{tabular}

Decrease of MTR

none

ADC

Increase of ADC

Angular gyrus
Superior occipital gyrus
Parahippocampal gyrus
Paracentral lobule
Inferior parietal gyrus
Superior parietal gyrus
Superior parietal gyrus
Postcentral gyrus
Precuneus
Middle temporal gyrus

right

2.33

0.012

right $\quad 2.05$

0.023

left

0.019

right

0.041

right $\quad 1.68 \quad 0.049$

left $\quad 2.65 \quad 0.005$

right $\quad 2.57 \quad 0.006$

left $\quad 1.75 \quad 0.043$

left $\quad 1.88 \quad 0.015$

right $\quad 2.22 \quad 0.049$

Decrease of ADC

Cerebellum, crus

left

2.13

0.019

Inferior frontal gyrus, opercular part

left

2.12

0.019

Inferior frontal gyrus, triangular part

left

left

0.030

Lingual gyrus

right

0.036

FA

0.025

Increase of FA

$\begin{array}{ccc}\text { right } & 2.75 & 0.004 \\ \text { right } & 1.70 & 0.047 \\ \text { right } & 1.81 & 0.038 \\ \text { left } & 2.06 & 0.022 \\ \text { left } & 1.74 & 0.044 \\ \text { right } & 2.43 & 0.009 \\ \text { left } & 2.23 & 0.015 \\ \text { right } & 1.93 & 0.029 \\ \text { left } & 1.83 & 0.037 \\ \text { right } & 2.06 & 0.022 \\ \text { left } & 2.13 & 0.019 \\ \text { right } & 2.25 & 0.014\end{array}$


Table 3 ROI analyses of grey matter volume, MTI, and DTI-values in patients with dystonia compared to those in healthy controls (group comparison) (Continued)

\begin{tabular}{lccl}
\hline Superior parietal gyrus & left & 2.90 & 0.002 \\
Superior parietal gyrus & right & 2.81 & 0.003 \\
Postcentral gyrus & left & 2.15 & 0.018 \\
Precentral gyrus & right & 1.76 & 0.042 \\
Precuneus & left & 2.39 & 0.010 \\
Precuneus & right & 1.73 & 0.044 \\
Supramarginal gyrus & right & 2.07 & 0.021 \\
Middle temporal gyrus & right & 2.23 & 0.015 \\
Cerebellum, vermis & & 1.77 & 0.041 \\
Cerebellum, vermis & & 1.96 & 0.028 \\
& & & \\
none & Decrease of FA & & \\
\hline
\end{tabular}

\section{MTR patients > controls}
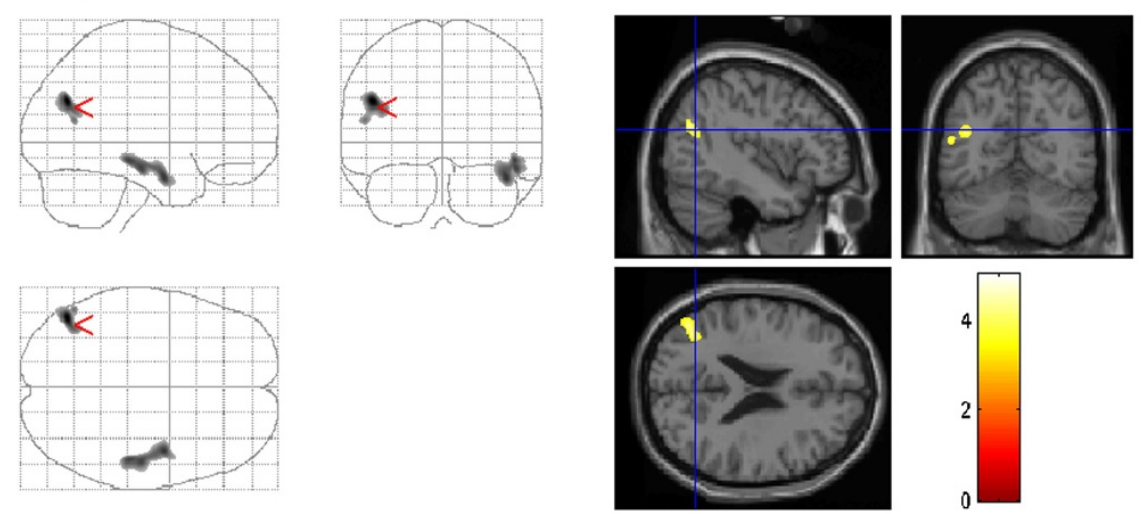

\section{MTR controls > patients}
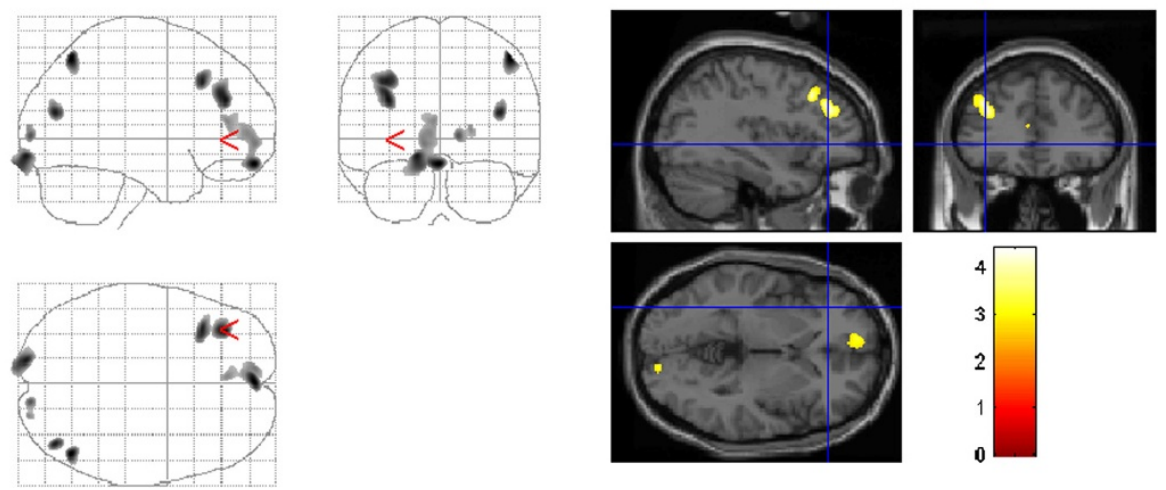

Figure 2 Changes of MTR in patients with dystonia compared to those in healthy controls (group comparison). Enhanced MTR was found in the left temporal medial gyrus, in the temporal lobe, in the secondary somatosensory cortex, and in the cingulate gyrus. MTR was reduced in the left dorsolateral prefrontal and frontal cortex (BA 9, 10,11) and in the primary and secondary visual cortex (BA 17, 19). 


\section{FA patients > controls}
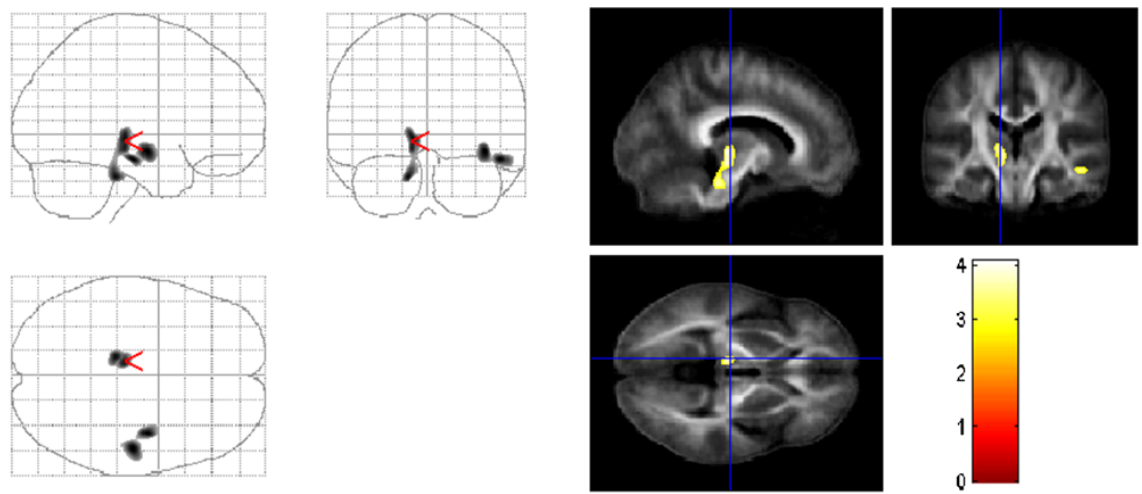

\section{FA controls > patients}
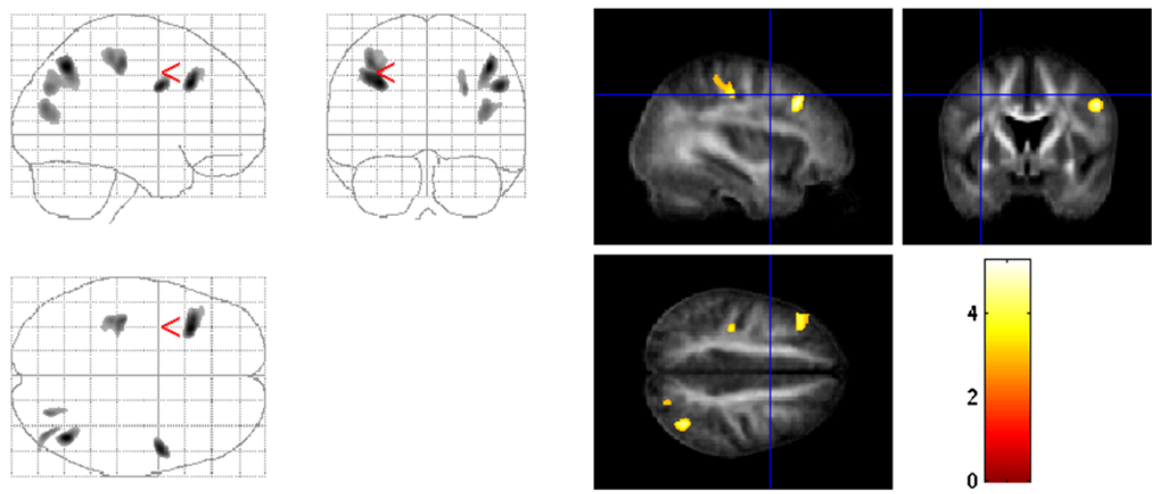

\section{ADC patients > controls}
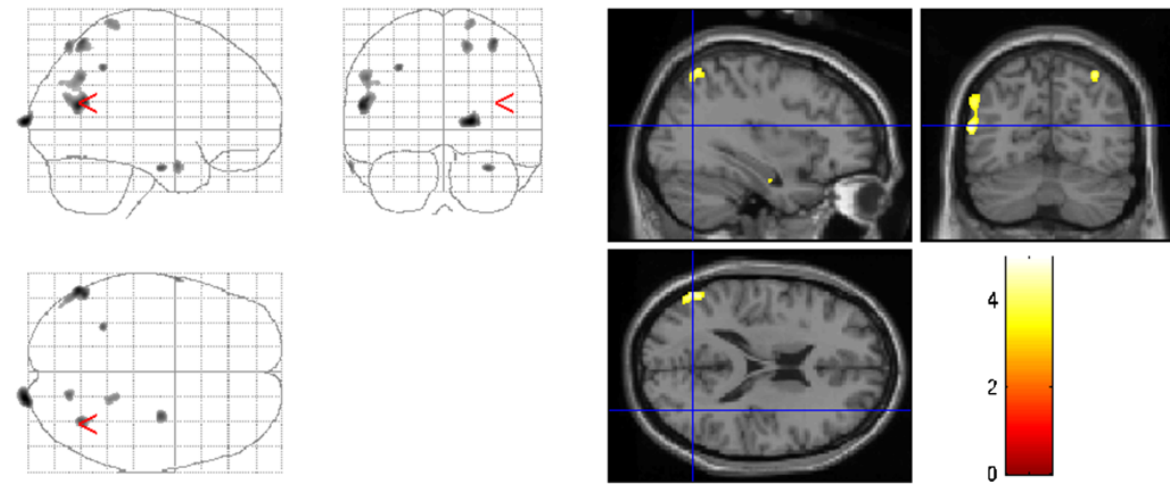

Figure 3 Changes of DTI-values in patients with dystonia compared to those in healthy controls (group comparison). FA was increased in brainstem, thalamus, and temporal areas. Decreased FA was observed in postcentral gyrus, frontal and occipital cortex. Diffusivity was enhanced in the area MT, and secondary somatosensory cortex.

[21]. Our study supports this view because we found structural changes in the basal ganglia, thalamus and brainstem.

We found a significant increase in grey matter in the putamen and pallidum. However, ROI analysis of both structures revealed no grey matter differences between the patients with dystonia and controls. Miscellaneous studies demonstrated grey matter increases in the putamen and internal globus pallidus [5,22,23]. However, these results are in contrast to a decrease in grey matter volume in the putamen in a smaller cohort of patients with dystonia [4] and in an ROI-based VBM study [16]. The discrepancy within all published morphometric studies on dystonia may be due to several methodological factors, among which the number of patients, 
Table 4 Changes of grey matter volume, MTI, and DTI-values in patients with dystonia and their correlation with clinical data

\begin{tabular}{|c|c|c|c|c|c|c|c|c|}
\hline \multirow[t]{2}{*}{ Brain region } & \multirow[t]{2}{*}{ Side } & \multirow[t]{2}{*}{ BA } & \multicolumn{3}{|c|}{ MNI space } & \multirow[t]{2}{*}{ t-value } & \multirow[t]{2}{*}{$P$ (uncorr) } & \multirow[t]{2}{*}{ Cluster size } \\
\hline & & & $\mathbf{x}$ & y & $\mathbf{z}$ & & & \\
\hline \multicolumn{9}{|c|}{ Positive correlation UDRS and grey matter volume } \\
\hline Frontal gyrus & right & 11 & 1 & 22 & -27 & 5.23 & 0.002 & 690 \\
\hline Cingulate gyrus & left & - & -12 & -19 & 31 & 4.81 & 0.004 & 310 \\
\hline Inferior frontal gyrus & left & - & -45 & 27 & 6 & 3.97 & 0.022 & 134 \\
\hline \multicolumn{9}{|c|}{ Positive correlation UDRS and FA } \\
\hline Middle occipital gyrus & right & 19 & 36 & -73 & 0 & 7.20 & $0.010^{*}$ & 1381 \\
\hline Cingulum, pars posterior & right & 30 & 13 & -65 & 8 & 5.00 & $0.001^{*}$ & 2258 \\
\hline Occipital lobe, cuneus & & 17 & 9 & -83 & 5 & 4.73 & & \\
\hline \multirow[t]{2}{*}{ Occipital lobe } & left & 19 & -40 & -66 & -16 & 5.02 & 0.006 & 200 \\
\hline & & 18 & -2 & -73 & -6 & 4.70 & 0.011 & 495 \\
\hline \multirow[t]{2}{*}{ Occipital lobe } & left & 19 & -36 & -83 & 4 & 4.27 & 0.024 & 416 \\
\hline & & & -27 & -82 & 2 & 3.92 & & \\
\hline \multirow[t]{3}{*}{ Frontal lobe } & left & 10 & -18 & 54 & 11 & 5.49 & $0.017^{*}$ & 1241 \\
\hline & & 32 & -23 & 40 & 8 & 4.32 & & \\
\hline & & 11 & -23 & 42 & -1 & 4.11 & & \\
\hline Frontal lobe & left & 8 & -23 & 18 & 37 & 4.92 & 0.007 & 386 \\
\hline Frontal lobe & right & 11 & 9 & 48 & -16 & 4.72 & 0.010 & 161 \\
\hline Parietal lobe, precuneus & left & 7 & -13 & -74 & 49 & 4.34 & 0.021 & 210 \\
\hline \multicolumn{9}{|c|}{ Negative correlation UDRS and ADC } \\
\hline \multirow[t]{3}{*}{ Occipital lobe, cingulum } & right & - & 19 & -62 & 10 & 5.48 & $0.014^{*}$ & 1531 \\
\hline & & & 20 & -73 & 14 & 4.20 & & \\
\hline & & & 28 & -71 & 18 & 4.02 & & \\
\hline Middle occipital gyrus & right & 19 & 38 & -72 & 5 & 4.14 & 0.026 & 412 \\
\hline \multicolumn{9}{|c|}{ Negative correlation disease duration and MTR } \\
\hline Middle frontal gyrus & left & 6 & -26 & 23 & 60 & 5.65 & 0.001 & 559 \\
\hline Precentral gyrus & left & 4 & -22 & -28 & 64 & 4.99 & 0.002 & 549 \\
\hline Fusiform gyrus & right & 37 & 46 & -56 & -21 & 4.97 & 0.002 & 687 \\
\hline \multicolumn{9}{|c|}{ Negative correlation disease duration and FA } \\
\hline Superior frontal gyrus & left & 8 & -26 & 20 & 54 & 5.89 & 0.001 & 255 \\
\hline Parietal lobe, precuneus & left & 7 & 19 & -72 & 50 & 5.19 & 0.004 & 356 \\
\hline Parietal lobe, precuneus & right & 7 & -4 & -72 & 44 & 4.32 & 0.022 & 135 \\
\hline Occipital lobe & left & 17 & -10 & -92 & -15 & 4.08 & 0.033 & 288 \\
\hline \multirow[t]{2}{*}{ Temporal lobe } & left & 37 & -44 & -45 & -13 & 4.15 & 0.029 & 278 \\
\hline & & & -40 & -53 & -10 & 3.79 & & \\
\hline \multicolumn{9}{|c|}{ Positive correlation disease duration and ADC } \\
\hline \multirow[t]{2}{*}{ Occipital gyrus } & right & 18 & 30 & -94 & -5 & 4.53 & 0.012 & 430 \\
\hline & & & 36 & -91 & -10 & 3.78 & & \\
\hline Occipital lobe. cuneus & Right & - & 5 & -99 & 4 & 4.16 & 0.025 & 246 \\
\hline \multicolumn{9}{|c|}{ Negative correlation disease duration and ADC } \\
\hline \multirow[t]{2}{*}{ Middle frontal gyrus } & left & 8 & -26 & 15 & 52 & 6.34 & $<0.001$ & 335 \\
\hline & & 6 & -41 & -2 & 49 & 4.74 & 0.008 & 118 \\
\hline Middle frontal gyrus & right & 6 & 25 & -12 & 64 & 4.26 & 0.021 & 118 \\
\hline
\end{tabular}


Table 4 Changes of grey matter volume, MTI, and DTI-values in patients with dystonia and their correlation with clinical data (Continued)

\begin{tabular}{|c|c|c|c|c|c|c|c|c|}
\hline \multirow[t]{2}{*}{ Inferior parietal lobule } & \multirow[t]{2}{*}{ right } & \multirow[t]{2}{*}{40} & 40 & -57 & 42 & 5.27 & \multirow[t]{2}{*}{0.003} & \multirow[t]{2}{*}{443} \\
\hline & & & 46 & -51 & 53 & 3.71 & & \\
\hline Inferior parietal lobule & right & 40 & 38 & -40 & 43 & 4.43 & 0.015 & 154 \\
\hline \multirow[t]{3}{*}{ Inferior parietal lobule } & \multirow[t]{3}{*}{ left } & \multirow[t]{3}{*}{40} & -50 & -40 & 47 & 5.15 & \multirow[t]{3}{*}{0.004} & \multirow[t]{3}{*}{825} \\
\hline & & & -40 & -41 & 43 & 4.66 & & \\
\hline & & & -44 & -33 & 42 & 4.03 & & \\
\hline Postcentral gyrus & right & 3 & 25 & -31 & 54 & 5.34 & 0.003 & 172 \\
\hline Postcentral gyrus & left & 3 & -22 & -30 & 63 & 4.80 & 0.008 & 614 \\
\hline \multirow[t]{2}{*}{ Precentral gyrus } & \multirow[t]{2}{*}{ right } & 4 & 55 & -10 & 45 & 4.55 & 0.012 & 382 \\
\hline & & 4 & 43 & -14 & 59 & 4.24 & 0.022 & 283 \\
\hline Parietal lobe, precuneus & left & 7 & -16 & -63 & 55 & 4.28 & 0.020 & 155 \\
\hline
\end{tabular}

* significant with FDR (false discovery rate).

disease duration and methods used for data analysis are the most important.

In another ROI-based analysis of the basal ganglia system, an increased FA in the putamen and a decreased $\mathrm{ADC}$ in the pallidum and putamen were found [8]. While FA remained unchanged, we also observed decreased ADC in the putamen of patients with dystonia. No alterations were found for MTI in the basal ganglia, suggesting that detected grey matter volume changes are not due to severe macromolecular matrix alterations or cellular damage. According to a previous study [22], there was no correlation between disease duration or UDRS with volumetric alterations in the basal ganglia. However, longitudinal studies are necessary to answer the question of whether these volume changes are to be regarded as primary structural changes or secondary adaptations [24].

\section{Brainstem and cerebellum}

Changes outside the basal ganglia can be interpreted to be a consequence of basal ganglia dysfunction. In a more recent view, dystonia can be regarded as a network disorder characterized by abnormal communication of any part of the network [25]. Among these networks, the cerebellum, cerebello-thalamo-cortical network and the pontine brainstem have received considerable attention $[20,26,27]$. A disruption of cerebellar output can be an important factor determining the occurrence of motor symptoms [27].

Evidence of abnormal interaction between the basal ganglia and cerebellar networks comes from structural and functional imaging studies and animal models. Our ROI analysis revealed cerebellar alterations in grey matter volume. Both increases and decreases in cerebellar grey matter volume were demonstrated before in VBM $[3,4]$. We also found altered MTI, diffusivity and FA values in the cerebellum of patients with dystonia. However, with regard to analysis of the cerebellum using DTI, several methodological limitations have to be taken into account, such as the low resolution after normalization and incomplete mapping of the cerebellum.

Decreased connectivity of the cerebellum with the thalamus was demonstrated by diffusion tractography in patients with clinically manifesting and non-manifesting hereditary dystonia [28]. Moreover, studies found increased metabolism in the cerebellum [28]. Animal models showing cerebellar dysfunction provide further support for the important role of the cerebellum in dystonia [27]. Our patients also exhibited DTI changes in the pontine brainstem, particularly the medial lemniscus, which carries sensory information from the gracile and cuneate nuclei to the thalamus. This is in line with DTI studies on hereditary dystonia, which revealed white matter changes in the dorsal pons [7,29]. The output from the basal ganglia to the brainstem targets regions that provide indirect input to the striatum [30]. It was postulated that these basal ganglia-brainstem pathways determine the degree of freedom of the automatic and volitional aspects of movements and are involved in the maintenance of arousal and attentive states and regulation of REM sleep [31].

\section{Somatosensory system and thalamus}

Although dystonia is characterized by motor problems, sensory abnormalities for temporal discrimination, kinesthesia, perception of vibratory tendon stimulation and somatotopy were frequently observed by special testing [32]. One possible reason for these sensory abnormalities can be a loss of lateral inhibition [21]. Our study underlines the involvement of the sensory system. Decreased grey matter volume and white matter changes were found in the primary and secondary somatosensory cortices. The observed decrease in grey matter volume in the secondary somatosensory cortex is in line with previous findings in 
patients with blepharospasm [22]. Our study showed a decreased FA in the primary and secondary somatosensory cortices, which was not demonstrable in previous ROI-based studies [8,9] or in a study of patients with hereditary dystonia [7].

Correlation analyses revealed dependency of these microstructural changes on disease duration, suggesting that DTI changes reflect adaptation processes in the somatosensory system during the disease course or because of botulinum toxin treatment. This is supported by the observation that a decrease in grey matter volume in the parietal lobe depends on the duration of botulinum toxin treatment in patients with blepharospasm [22]. Likely, botulinum toxin leads to remodeling processes in the somatosensory system because of its local denervation in the muscle [33]. On the other hand, this toxin can mask morphological changes associated with dystonia $[9,34]$. To address this issue, a correlation analysis of the duration and dose of botulinum toxin should be performed.

DTI changes in the fibre tracts connecting the primary sensorimotor areas with the subcortical structures may be the basis for the impairment of GABAergic cortical inhibition proposed as a pathophysiological feature of dystonia $[7,21]$. Furthermore, changes in DTI in the thalamus were demonstrable, showing that the thalamus exhibits not only functional $[35,36]$ but also structural alterations in patients with dystonia. Under the view of dystonia as a network disease, this result is not surprising because the basal ganglia not only contacts regions of the thalamus that project directly back to the basal ganglia input nuclei but also project back to those cortical regions providing original inputs to the striatum [30].

In summary, our results strongly support the thesis that the sensory system is frequently involved in dystonia [34,37-40]. In addition, this provides further evidence for the concept that abnormal motor learning or abnormal plasticity may play a crucial role in dystonia $[19,21]$.

\section{Frontal areas}

A decrease in grey matter and microstructural changes in white matter were observed in the supplementary motor area and primary motor cortex. Other prefrontal areas (BA 10) showed enhanced grey matter volume and decreased MTR. MTR was increased in the anterior cingulum of the patients with dystonia. While grey matter atrophy in the supplementary motor area has been previously demonstrated [3], this is the first time that an enhanced FA was observed in the supplementary motor area of patients with dystonia, supporting that these changes are not only functional [41,42] but also structural. The supplementary motor area is responsible for planning complex movements; therefore, it is connected with the primary motor cortex and the thalamus, both of which showed structural changes in this study. While a grey matter decrease in the supplementary motor area was not demonstrable in smaller cohorts of patients with dystonia [3] or blepharospasm [22], the decreased FA is in line with previous findings [7].

It seems reasonable that alterations in the dorsolateral prefrontal cortex can be observed in patients with dystonia because this structure is connected to the orbitofrontal cortex, thalamus, basal ganglia, hippocampus and primary and secondary association areas, including the posterior temporal, parietal and occipital areas. In a study of regional cerebral blood flow in 6 patients with idiopathic torsion dystonia, patients showed significant overactivity in the ipsilateral dorsolateral prefrontal cortex, demonstrating inappropriate activity of striatofrontal projections and impaired activity of motor executive areas [41]. Here, involvement of the dorsolateral prefrontal cortex could be demonstrated by DTI and MTI changes in the patients with dystonia and was supported by previous volumetric [3] and functional imaging studies [35,41]. With regard to the grey matter increase in the prefrontal areas (BA 10), it seems that the decreased MTR in this region is not caused by neuronal cell loss. The correlation between changes in the primary or secondary motor cortex and disease duration suggests that these are secondary adaptations, probably caused by modified thalamic firing patterns. This is supported by the positive correlation between the severity of dystonia (UDRS) and increase in grey matter volume in the frontal gyrus and cingulum.

\section{Visual system}

Because the basal ganglia and motor areas are heavily connected with visual areas, one can assume that abnormalities in other sensory modalities, such as visual, may also be implicated in dystonia. We frequently observed changes in the lateral and medial surfaces of the occipital lobe, the frontal eye field and area MT with all three imaging methods. The frontal eye field in the patients with dystonia showed enhanced grey matter volume. In the secondary visual cortex, decreased MTR and FA and an increased diffusivity were found. In the visual areas, FA decreased (primary and secondary visual area, frontal eye field) and diffusivity increased (secondary visual cortex) with disease duration. With increasing severity of dystonia (UDRS), FA increased in the primary and secondary visual areas and the frontal eye field, whereas diffusivity decreased in the secondary visual cortex. Evidence for alterations in the visual system came from neuropsychological studies [43] and one volumetric analysis showing a grey matter decrease in the visual cortex [3]. The severity of dystonia (UDRS) correlated with increased FA in the primary and secondary cortices and decreased diffusivity in the secondary visual cortex. Disease duration correlated with decreased FA in the primary and secondary cortices and the frontal eye field. 
Moreover, duration correlated with increased diffusivity in the secondary visual cortex and decreased diffusivity in the frontal eye field. These results probably display the adaptation to the dystonic head shift and show some similarity to the grey matter volume increase in MT after juggling training [44].

The present study provides evidence that dystonia is a complex disturbance of neuronal circuits involving the basal ganglia, thalamus, motor cortex, premotor area and visual and frontal cortices.

\section{Methodological aspects and limitations of the study}

This study is not free of limitations. The sample size is comparable with those in other studies on dystonia; however, analysis of more patients would be useful in order to study the impact of several clinical variables. Moreover, to understand the nature of primary and secondary processes, a longitudinal assessment of patients would be a challenge to expand the results obtained via correlation analysis.

As in many imaging studies, a primary limitation is that most data were uncorrected. Analyses using uncorrected voxel-based statistics have increased sensitivity to detect particularly small abnormalities, but the number of false abnormal regions is higher than that in analyses using corrected voxel-based statistics [45]. Because the usefulness of SVC described in the methods section is controversial, we also used ROI analyses with anatomically predefined regions for the group comparison; this supported and augmented the results from voxel-based imaging. In terms of DTI, both FA and ADC values were changed in the patients with dystonia. The value that accurately reflects white matter damage is not yet clear. Discrepancies in the DTI studies are primarily caused by differences in the number and age of the patients and severity and duration of the disease. One crucial factor is also the dataset. A dataset with more gradient directions was more sensitive to decreases in FA, while the dataset with more $b$ values was more sensitive to increases in mean diffusivity [46]. Last, the results are influenced by the choice of MRI scanner (3-T or 1.5-T). A higher field strength at $3.0 \mathrm{~T}$ would probably have great impact on the results for DTI scalars and MTI.

\section{Conclusions}

Despite the discrepancies in imaging results, some unifying conclusions can be drawn from the results of this study. VBM, DTI and MTI revealed widespread structural changes that were not restricted to the basal ganglia. Structural alterations were observed in the thalamus, motor cortex, premotor and frontal cortices, visual system and cerebellum of the patients with dystonia. This study provides further evidence that cervical dystonia is a multisystem disease that involves several networks such as the motor, sensory and visual systems.

\section{Abbreviations}

BA: Brodman area; DTI: Diffusion tensor imaging; MRI: Magnetic resonance imaging; MTI: Magnetization transfer imaging; MTR: Magnetization transfer ratio; PD: Proton density; VBM: Voxel-based morphometry.

\section{Competing interests}

The authors declare that they have no competing interests.

\section{Authors' contributions}

Conceived and designed the experiments: ThP, JG, RD. Performed the experiments: ThP, JG, BK, MB, HB. Analyzed the data: ThP, JG, BK, TP, AG. Wrote the paper: TP. All authors read and approved the final manuscript.

\section{Acknowledgements}

We thank Heike Gorzolla for coordinating of patient recruitment and Andreas Niesel for technical support.

\section{Funding}

Tino Prell reports received funding funding in the form of an IZKF grant from University Hospital Jena.

Thomas Peschel reports no disclosures.

Bernadette Köhler reports no disclosures.

Martin Bokemeyer reports no disclosures.

Reinhard Dengler reports no disclosures in regard to the current study. RD received honoraria, research grants and travel grants from PharmAllergan, Ipsen Pharma, Merz Pharma, Boehringer-Ingelheim and Bayer Health Care. Albrecht Günther received funding for travel and/or speaker honoraria from Merz, IPSEN, Boehringer-Ingelheim (2007-2011).

Julian Grosskreutz received funding from BMBF (the Bundesministerium für Bildung und Forschung) grant to JG (ERMCC-NDEG) within the framework of the ERANET-NEURON program of the European Union.

\section{Author details}

${ }^{1}$ Hans-Berger Department of Neurology, Jena University Hospital, Erlanger Allee 101, 07747 Jena, Germany. ${ }^{2}$ Department of Psychiatry and Psychotherapy, Medical School Hannover, Carl-Neuberg-Strasse 1, 80625 Hannover, Germany. ${ }^{3}$ Department of Neurology and Clinical Neurophysiology, Medical School Hannover, Carl-Neuberg-Str.1, 30625 Hannover, Germany. ${ }^{4}$ Department of Neuroradiology, Jena University Hospital, Erlanger Allee 101, 07747 Jena, Germany. ${ }^{5}$ Center for Sepsis Control and Care, Jena University Hospital, Erlanger Allee 101, 07747 Jena, Germany.

Received: 24 June 2013 Accepted: 9 October 2013

Published: 16 October 2013

\section{References}

1. Defazio G, Abbruzzese G, Livrea P, Berardelli A: Epidemiology of primary dystonia. Lancet Neurol 2004, 3:673-678.

2. Chan J, Brin MF, Fahn S: Idiopathic cervical dystonia: clinical characteristics. Mov Disord 1991, 6:119-126.

3. Draganski B, Thun-Hohenstein C, Bogdahn U, Winkler J, May A: "Motor circuit" gray matter changes in idiopathic cervical dystonia. Neurology 2003, 61:1228-1231.

4. Obermann M, Yaldizli O, De Greiff A, Lachenmayer ML, Buhl AR, Tumczak F, Gizewski ER, Diener HC, Maschke M: Morphometric changes of sensorimotor structures in focal dystonia. Mov Disord 2007, 22:1117-1123.

5. Egger K, Mueller J, Schocke M, Brenneis C, Rinnerthaler M, Seppi K, Trieb T, Wenning GK, Hallett M, Poewe W: Voxel based morphometry reveals specific gray matter changes in primary dystonia. Mov Disord 2007, 22:1538-1542.

6. Ramdhani RA, Simonyan K: Primary dystonia: conceptualizing the disorder through a structural brain imaging lens. Tremor Other Hyperkinet Mov (N Y) 2013, 3. http://tremorjournal.org/article/view/152.

7. Carbon M, Kingsley PB, Su S, Smith GS, Spetsieris P, Bressman S, Eidelberg D: Microstructural white matter changes in carriers of the DYT1 gene mutation. Ann Neurol 2004, 56:283-286.

8. Colosimo C, Pantano P, Calistri V, Totaro P, Fabbrini G, Berardelli A: Diffusion tensor imaging in primary cervical dystonia. J Neurol Neurosurg Psychiatry 2005, 76:1591-1593. 
9. Fabbrini G, Pantano P, Totaro P, Calistri V, Colosimo C, Carmellini M, Defazio G, Berardelli A: Diffusion tensor imaging in patients with primary cervical dystonia and in patients with blepharospasm. Eur J Neurol 2008, 15:185-189.

10. Bonilha L, de Vries PM, Vincent DJ, Rorden C, Morgan PS, Hurd MW, Besenski N, Bergmann KJ, Hinson VK: Structural white matter abnormalities in patients with idiopathic dystonia. Mov Disord 2007, 22:1110-1116.

11. Eckert T, Sailer M, Kaufmann J, Schrader C, Peschel T, Bodammer N, Heinze HJ, Schoenfeld MA: Differentiation of idiopathic Parkinson's disease, multiple system atrophy, progressive supranuclear palsy, and healthy controls using magnetization Transfer Imaging. Neuroimage 2004, 21:229-235.

12. Rademacher J, Engelbrecht V, Burgel U, Freund H, Zilles K: Measuring in vivo myelination of human white matter fiber tracts with magnetization transfer MR. Neuroimage 1999, 9:393-406.

13. Woods RP, Mazziotta JC, Cherry SR: MRI-PET registration with automated algorithm. J Comput Assist Tomogr 1993, 17:536-546.

14. Grosskreutz J, Kaufmann J, Fradrich J, Dengler R, Heinze HJ, Peschel T: Widespread sensorimotor and frontal cortical atrophy in Amyotrophic Lateral Sclerosis. BMC Neurol 2006, 6:17.

15. Camara E, Bodammer N, Rodriguez-Fornells A, Tempelmann C: Age-related water diffusion changes in human brain: a voxel-based approach. Neuroimage 2007, 34:1588-1599.

16. Pantano P, Totaro P, Fabbrini G, Raz E, Contessa GM, Tona F, Colosimo C, Berardelli A: A transverse and longitudinal MR imaging voxel-based morphometry study in patients with primary cervical dystonia. AJNR Am J Neuroradiol 2011, 32:81-84.

17. Schiffer B, Peschel T, Paul T, Gizewski E, Forsting M, Leygraf N, Schedlowski M, Krueger TH: Structural brain abnormalities in the frontostriatal system and cerebellum in pedophilia. J Psychiatr Res 2007, 41:753-762.

18. Tzourio-Mazoyer N, Landeau B, Papathanassiou D, Crivello F, Etard O, Delcroix N, Mazoyer B, Joliot M: Automated anatomical labeling of activations in SPM using a macroscopic anatomical parcellation of the MNI MRI single-subject brain. Neuroimage 2002, 15:273-289.

19. DeLong MR, Wichmann T: Circuits and circuit disorders of the basal ganglia. Arch Neurol 2007, 64:20-24

20. Filip P, Lungu OV, Bares M: Dystonia and the cerebellum: a new field of interest in movement disorders? Clin Neurophysiol 2013, 124:1269-1276.

21. Hallett M: Neurophysiology of dystonia: the role of inhibition. Neurobiol Dis 2011, 42:177-184.

22. Etgen T, Muhlau M, Gaser C, Sander D: Bilateral grey-matter increase in the putamen in primary blepharospasm. I Neurol Neurosurg Psychiatry 2006, 77:1017-1020.

23. Black KJ, Ongur D, Perlmutter JS: Putamen volume in idiopathic focal dystonia. Neurology 1998, 51:819-824.

24. Vitek JL: Pathophysiology of dystonia: a neuronal model. Mov Disord 2002, 17(Suppl 3):S49-S62.

25. Neychev VK, Gross RE, Lehericy S, Hess EJ, Jinnah HA: The functional neuroanatomy of dystonia. Neurobiol Dis 2011, 42:185-201.

26. Blood AJ, Kuster JK, Woodman SC, Kirlic N, Makhlouf ML, Multhaupt-Buell TJ, Makris N, Parent M, Sudarsky LR, Sjalander G, et al: Evidence for altered basal ganglia-brainstem connections in cervical dystonia. PLoS One 2012, 7:e31654.

27. Lehericy S, Tijssen MA, Vidailhet M, Kaji R, Meunier S: The anatomical basis of dystonia: current view using neuroimaging. Mov Disord 2013, 28:944-957.

28. Niethammer M, Carbon M, Argyelan M, Eidelberg D: Hereditary dystonia as a neurodevelopmental circuit disorder: evidence from neuroimaging. Neurobiol Dis 2011, 42:202-209.

29. Carbon M, Kingsley PB, Tang C, Bressman S, Eidelberg D: Microstructural white matter changes in primary torsion dystonia. Mov Disord 2008, 23:234-239.

30. Redgrave P, Coizet V: Brainstem interactions with the basal ganglia. Parkinsonism Relat Disord 2007, 13(Suppl 3):S301-S305.

31. Takakusaki K, Saitoh K, Harada H, Kashiwayanagi M: Role of basal gangliabrainstem pathways in the control of motor behaviors. Neurosci Res 2004 50:137-151

32. Tinazzi M, Fiorio M, Fiaschi A, Rothwell JC, Bhatia KP: Sensory functions in dystonia: insights from behavioral studies. Mov Disord 2009, 24:1427-1436.

33. Draganski B, Moser T, Lummel N, Ganssbauer S, Bogdahn U, Haas F, May A: Decrease of thalamic gray matter following limb amputation. Neuroimage 2006, 31:951-957.

34. Blood AJ, Tuch DS, Makris N, Makhlouf ML, Sudarsky LR, Sharma N: White matter abnormalities in dystonia normalize after botulinum toxin treatment. Neuroreport 2006, 17:1251-1255.
35. Detante O, Vercueil L, Thobois S, Broussolle E, Costes N, Lavenne F, Chabardes S, Lebars D, Vidailhet M, Benabid AL, et al: Globus pallidus internus stimulation in primary generalized dystonia: a H215O PET study. Brain 2004, 127:1899-1908.

36. Eidelberg D, Moeller JR, Antonini A, Kazumata K, Nakamura T, Dhawan V, Spetsieris $P$, deLeon $D$, Bressman SB, Fahn S: Functional brain networks in DYT1 dystonia. Ann Neurol 1998, 44:303-312.

37. Bara-Jimenez $W$, Shelton $\mathrm{P}$, Hallett $\mathrm{M}$ : Spatial discrimination is abnormal in focal hand dystonia. Neurology 2000, 55:1869-1873.

38. Tinazzi M, Rosso T, Fiaschi A: Role of the somatosensory system in primary dystonia. Mov Disord 2003, 18:605-622.

39. Wu CC, Fairhall SL, MCNair NA, Hamm JP, Kirk IJ, Cunnington R, Anderson T, Lim VK: Impaired sensorimotor integration in focal hand dystonia patients in the absence of symptoms. J Neurol Neurosurg Psychiatry 2010, 81:659-665.

40. Opavsky R, Hlustik P, Otruba P, Kanovsky P: Sensorimotor network in cervical dystonia and the effect of botulinum toxin treatment: a functional MRI study. J Neurol Sci 2011, 306:71-75.

41. Ceballos-Baumann AO, Passingham RE, Warner T, Playford ED, Marsden CD, Brooks DJ: Overactive prefrontal and underactive motor cortical areas in idiopathic dystonia. Ann Neurol 1995, 37:363-372.

42. Dresel C, Haslinger B, Castrop F, Wohlschlaeger AM, Ceballos-Baumann AO: Silent event-related fMRI reveals deficient motor and enhanced somatosensory activation in orofacial dystonia. Brain 2006, 129:36-46.

43. Hinse $P$, Leplow B, Humbert $T$, Lamparter U, Junge A, Emskotter T: Impairment of visuospatial function in idiopathic spasmodic torticollis. J Neurol 1996, 243:29-33.

44. Draganski B, Gaser C, Busch V, Schuierer G, Bogdahn U, May A: Neuroplasticity: changes in grey matter induced by training. Nature 2004, 427:311-312.

45. Radua J, Canales-Rodriguez EJ, Pomarol-Clotet E, Salvador R: Validity of modulation and optimal settings for advanced voxel-based morphometry. Neuroimage 2013. doi:10.1016/j.neuroimage.2013.07.084.

46. Rae CL, Correia MM, Altena E, Hughes LE, Barker RA, Rowe JB: White matter pathology in Parkinson's disease: the effect of imaging protocol differences and relevance to executive function. Neuroimage 2012 62:1675-1684.

doi:10.1186/1471-2202-14-123

Cite this article as: Prell et al: Structural brain abnormalities in cervical dystonia. BMC Neuroscience 2013 14:123.

\section{Submit your next manuscript to BioMed Central and take full advantage of:}

- Convenient online submission

- Thorough peer review

- No space constraints or color figure charges

- Immediate publication on acceptance

- Inclusion in PubMed, CAS, Scopus and Google Scholar

- Research which is freely available for redistribution 